\title{
Pilot study and key feasibility factors of a staff training intervention and reduction of antipsychotic prescription practice in Mexican urban care homes: study protocol.
}

\author{
Sara Torres-Castro ${ }^{1 *}$, Mariana López-Ortega ${ }^{1}$, Adrián Martínez-Ruiz ${ }^{1}$, Luis Miguel Gutiérrez-Robledo ${ }^{1}$, \\ Azucena Guzmán². \\ ${ }^{1}$ Instituto Nacional de Geriatría, Mexico City, Mexico \\ ${ }^{2}$ Clinical Psychology Department, School of Health in Social Science, University of Edinburgh, UK
}

\begin{abstract}
Background: In a context of rapid population ageing and increase in chronic illnesses including dementia in Mexico, there is a need to develop long-term care strategies in order to improve the quality of life, people affected by dementia and the people that care for them. In 2015, the prevalence of dementia in Mexico was $6.1 \%$ and it is estimated to reach to over 1.5 million by the year 2030, posing a great challenge to formal and informal caregivers. In 2014, Mexico developed a Dementia Strategy National Plan (Plan de Acción Alzheimer y otras demencias) and the objectives eight and nine of it aims to train the care work force on non-pharmacological and health professionals in care homes, and improve the appropriate antipsychotic prescription to treat challenging behavior respectively. Previous UK-based studies have been successful in training staff and health professionals by optimizing the prescription of antipsychotic medication and by implementing psychosocial interventions to treat behavioral and psychological symptoms associated to dementia.
\end{abstract}

Objective: The aim of this study was to develop PROCUIDA-Demencia (in Spanish: Programa para el Cuidado Optimo en Demencia), a staff training package.

Methods: A randomized controlled mixed methods feasibility study in eight care homes $(\mathrm{N}=\mathbf{5 0 0}$ beds approximately). The goal of the study is to investigate a set of interventions to promote psychosocial activities and review antipsychotic medication to treat Behavioral and Psychological Symptoms of dementia. This is a twelve weeks and follow up study that involves the development of the staff training package for Mexican care homes (PROCUIDA-Demencia) based on two arms: Staff Training with PROCUIDA-Demencia vs Treatment as Usual (TAU).

Conclusions: If feasible, PROCUIDA-Demencia Staff Training Model could be developed in a larger an easy-to-use set of therapies that benefit cognition, behavior, mood and quality of life in individuals with Dementia, and to staff in care homes across Mexico. It is expected that the results will provide the base for establishing a larger randomised control trial.

Keywords: Dementia, Antipsychotic medication, Person-centred care, Behavior, Staff-training, Psychosocial interventions, Care homes.

\begin{abstract}
Abbreviations: ACE-III: Addenbrooke's Cognitive Examination Spanish version; ADI: Alzheimer Disease International; ADQ: Approaches to Dementia Questionnaire; BPSD: Behavioral and Psychological Symptoms of Dementia; CDR: Clinical Dementia Rating; DANCIN: Psychomotor Dance Therapy Intervention; DSM-V: Diagnosis of neurodegenerative disease; GP: General practitioner; INGER: National Institute of Geriatrics; MHAS: Mexican Health and Aging Study; NICE: National Institute for Clinical Excellence; NPI-
\end{abstract}

NH: Neuropsychiatric Inventory Nursing Home Spanish Version; PCC: Person-Centre Care Therapy; PI: Principal investigator; PROCUIDA-Demencia: Programmed for Optimizing Care in Dementia; QoL-AD: Quality of LifeAlzheimer's Disease; RAM: Review of Antipsychotic Medication; SCIDS: Sense of Competence in Dementia Care Staff; SIGN: Scottish Intercollegiate Guidelines Network; SPPB: Short Physical Performance Battery; STATA: Data Analysis and Statistical Software; TAU: Treatment as Usual; WHELD: Model Dementia Champion. 
Citation: Torres-Castro S, López-Ortega M, Martínez-Ruiz A, et al. Pilot study and key feasibility factors of a staff training intervention and reduction of antipsychotic prescription practice in Mexican urban care homes: study protocol. J Ment Health Aging. 2018;2(1):47-55.

\section{Introduction}

It has been estimated that in 2015 about 46.8 million people worldwide were living with dementia. Also, because of the rapid aging population in low-and middle-income countries, it has been reported that this number will exponentially increase every 20 years, reaching 74.7 million in 2030 and 131.5 million in 2050 [1,2]. The Alzheimer Disease International association (ADI) highlights the importance of prioritizing research as population ages, in these countries.

In high-income countries, research has concentrated in developing a more adequate care strategies focused in people with dementia and in those who care for them. In the UK, the National Institute for Clinical Excellence (NICE) and the Scottish Intercollegiate Guidelines Network (SIGN) have emphasized the development of non-drug interventions, care staff interventions and better outcome measurement [3] to improve quality of care.

Mexico is experiencing a rapid ageing population process. By 2015 the population aged 60 years and older represented 10.4 per cent of total population and it is expected to represent 14.8 per cent in 2030 [4]. Accordingly to the studies conducted by the 10/66 Dementia Research Group the prevalence of dementia in Mexico reaches $7.4 \%$ for urban and $7.3 \%$ for rural areas [5]. Moreover, the Mexican Health and Aging Study (MHAS) found that the prevalence of dementia in Mexican population was $6.1 \%$ in subjects 60 years and older, and the incidence rate was 27.3 per 1,000 person-years [6]. It is expected that by 2030 the number of people affected with dementia in Mexico will rise from 800,000 in 2015 to 1.5 million [1].

Research in Ageing Studies has been increasing in Mexico for the last decades, this is due the availability to conduct longitudinal studies, and community based national surveys and qualitative studies. However, there is a lack of intervention studies on formal and informal health care scenarios. Formal long-term care policies aimed to attend the older people with dementia are still needed in Mexico. Few public institutions are available for these purposes. Furthermore many private and community based institutions comprised a wide range of settings and quality of care. A legal framework related to social care is in place but does not enhance long-term care and there is neither national, state, and local compulsory registration system nor mandatory standard of care. Consequently, there is an imprecise knowledge regarding the total number of care homes and models of care accountable to trend older adults with dementia [7].

In Mexico, the National Institute of Geriatrics (INGER) has a multidisciplinary approach to dementia. It has two main goals regarding dementia approach: 1) To implement models of care specifically for older population and also implement education in private and public health sectors, and 2) To establish a health care model for older adults with and without cognitive impairment. Furthermore, in 2014 Mexico developed a Dementia Strategy Plan; it has been supported by the Mexican National Health System. The Plan aims to promote the well-being of people affected by dementia and their families, it also aims to improve the quality of care delivered by care workers and health professionals in care homes, and improve the appropriate antipsychotic prescription to treat challenging behavior [8].

A number of studies have evaluated the effectiveness of staff training throughout a range of learning strategies, coaching, supervision and role modeling technics $[9,10]$. In the UK, the model "WHELD-Dementia Champion" [1113] consists of coaching and training staff on reviewing antipsychotics and implementing psychosocial interventions. WHELD programme has shown significant results on behavioral improvement and quality of life [13]. Likewise, models for better supervision in the form of an 'interactive staff training approach' [14-16] and strategies on how to provide feedback [17] can potentially be adapted to facilitate the learning and development process. Also, maintaining effective communication with the care home manager, and staff nomination during the investigation process, have been shown to have similar results [18].

Care staff training research has found that if training is provided, then the quality of life, the cognitive stimulation and physical activity of residents are increased [19,20]. Furthermore, it has been reported that if incentives and recognition are given to staff for learning new skills, then the professional performance also improves. The Programmed for Optimizing Care in Dementia (PROCUIDA-Demencia) study is part of the action plan to support the Dementia Strategy Plan in Mexico. It does it particularly by optimizing the prescription of antipsychotic medication and by training the work force to implement psychosocial interventions in order to treat stress and supporting the workforce in longterm care.

\section{Method}

\section{Study objectives}

This is a feasibility study of the PROCUIDA-Demencia program when provided in care homes for older adults affected by dementia. This study has been designed around the Medical Research Council framework for complex interventions [21] completing the cycle of evidence/theory/ modeling, development, feasibility/piloting, evaluation, and dissemination/implementation. It is hypothesized that PROCUIDA-Demencia will provide a vehicle for staff development, which will enhance the quantity and quality of care home staff-resident interactions.

\section{Study design}

A randomized controlled mixed methods feasibility study in eight care homes will be used for this study ( $\mathrm{N}=500$ beds approximately). It will involve two groups: PROCUIDADemencia and Treatment as Usual (TAU), participants will be randomly assigned either to PROCUIDA-Demencia or 
to Treatment as Usual (TAU) group, with a minimum of 25 participants with dementia and a minimum of 25 staff in each group. Care Homes will invite available and committed staff to complete a three day training given by UK/ International partner and will achieve satisfactory fidelity to the implementation of the interventions. Research assistants blind to group allocation will collect all the outcome measures data at baseline, 12 weeks and 24 weeks. Staff focus groups with semi-structured interviews regarding the experience of taking part in the interventions will be conducted. Also, semi-structured interviews will be conducted with homemanagers (at baseline and at the end of the study). The costanalysis data will be acquired by applying already developed questionnaires.

\section{Number of participants and statistical power}

A formal power calculation is not appropriate because this is a feasibility study; therefore, analysis of intervention efficacy is not the study objective.

\section{Randomization}

Care homes randomization will receive either PROCUIDA-Demencia or Treatment as Usual (TAU) using secure web access to the remote randomization centre at National Institute of Geriatrics. The randomization will be performed by dynamic allocation [22]. Control groups will receive the training and activities material at the end of the study. In this study, the outcome evaluators and data analysts will be blinded.

\section{Qualitative evaluation}

The Focus Groups data will be transcribed verbatim. The analysis will be made across the three groups: staff, residents and relatives through cross-interconnected stages 'framework analysis' [23]. We will develop themes and sub-themes of the participants' experiences and views of PROCUIDA-Demencia. This method has seven distinct phases that are interlinked and form a methodological and rigorous framework [24]. For example, data will be analyzed

Table 1. Screening and Outcomes Measures.

\begin{tabular}{|c|c|c|c|}
\hline Scales & Description & Measures & Application stage \\
\hline \multicolumn{4}{|c|}{ Screening Measures } \\
\hline \multicolumn{4}{|c|}{ Residents } \\
\hline $\begin{array}{l}\text { Short Physical Performance } \\
\text { Battery (SPPB) (Guralnik et } \\
\text { al. 1994) [25] }\end{array}$ & $\begin{array}{l}\text { This version contains balance, gait, strength and endurance sections such that } \\
\text { combines scores provide a risk of falls assessment. Five minutes to administer }\end{array}$ & Assess mobility & $\begin{array}{l}\text { Screening inclusion } \\
\text { criteria for residents }\end{array}$ \\
\hline $\begin{array}{l}\text { Clinical Dementia Rating } \\
\text { (CDR) (Morris, 1993) [26] }\end{array}$ & $\begin{array}{l}\text { Using a structured interview, six domains are assessed in terms of a person's cognitive } \\
\text { and functional performance. Including memory, orientations, judgment, problem solving, } \\
\text { community affairs, home and hobbies and personal care. CDR scores in each of these } \\
\text { domains are combined to obtain a composite score ranging from } 0 \text { 'none' through } 3 \\
\text { 'severe' } 20 \text { minutes to administer. }\end{array}$ & $\begin{array}{l}\text { Used to quantify } \\
\text { the severity } \\
\text { offstage of } \\
\text { dementia } \\
\text { diagnosis. }\end{array}$ & $\begin{array}{l}\text { Screening inclusion } \\
\text { criteria for residents }\end{array}$ \\
\hline \multicolumn{4}{|c|}{ Outcome Measures } \\
\hline \multicolumn{4}{|c|}{ Residents } \\
\hline $\begin{array}{l}\text { Quality of Life-Alzheimer's } \\
\text { Disease (QoL-AD) (Rosas- } \\
\text { Carrasco et al. 2010) [27] }\end{array}$ & $\begin{array}{l}\text { A brief } 13 \text {-item measure, with scores ranging from } 13 \text { to } 52 \text {. It is designed for use } \\
\text { by dementia patients and caregivers, and includes assessments of the individual's } \\
\text { relationships with friends and family, overall assessment of life quality, and concerns } \\
\text { about finances, physical condition and mood. Qol-AD for quality of life. }\end{array}$ & Quality of Life & $\begin{array}{l}\text { Baseline (before } \\
\text { intervention) Follow-up } \\
\text { week } 12 \text { and } 24 .\end{array}$ \\
\hline $\begin{array}{l}\text { Neuropsychiatric Inventory } \\
\text { Nursing Home Spanish } \\
\text { Version (NPI-NH) Boada et } \\
\quad \text { al. } 2005 \text { [29] }\end{array}$ & $\begin{array}{l}\text { The NPI-NH is a validated structured interview assessment with the informant (care } \\
\text { staff) that assesses behavioural disturbances in the residents with Dementia. This } \\
\text { version contains ten behavioural and two neurodegenerative areas. The frequency, } \\
\text { severity and care staff distress levels for each domain are measured. The total possible } \\
\text { maximum score is } 144 \text {. A higher score reflects increased frequency and severity of the } \\
\text { disturbances. } 15-20 \text { minutes to administer. }\end{array}$ & Behaviour & $\begin{array}{l}\text { Baseline (before } \\
\text { intervention) Follow-up } \\
\text { week } 12 \text { and } 24 .\end{array}$ \\
\hline \multicolumn{4}{|c|}{ Staff } \\
\hline $\begin{array}{l}\text { Maslach Burnout Inventory } \\
\text { (MBI). Maslach C, et al. } \\
1981[30]\end{array}$ & $\begin{array}{c}\text { It includes five general scales: i) emotional exhaustion: feelings of exhaustion by } \\
\text { one's work; ii) depersonalization: measures of unfeeling and impersonal response } \\
\text { toward recipients of one's service, iii) care treatment or instruction; iv) personal } \\
\text { accomplishment: measures feelings of competence and v) successful achievement in } \\
\text { one's work. }\end{array}$ & Burnout & $\begin{array}{l}\text { Baseline (before } \\
\text { intervention) Follow-up } \\
\text { week } 12 \text { and } 24 .\end{array}$ \\
\hline $\begin{array}{l}\text { Sense of Competence } \\
\text { in Dementia Care Staff } \\
\text { (SCIDS) (no-standardised } \\
\text { in Spanish translation). } \\
\text { Schepers et al. 2012 [32] }\end{array}$ & $\begin{array}{c}\text { A self-report measure comprises } 17 \text { items across four subscales (building relationships, } \\
\text { professionalism, care challenges and maintaining personhood). Each item is rated on } \\
\text { a four-point scale from } 1 \text { 'Not at all' to } 4 \text { 'Very much'. A total score (/68) and individual } \\
\text { subscale scores can be calculated. Higher scores represent a higher level of sense of } \\
\text { competence }\end{array}$ & $\begin{array}{l}\text { Assess the care } \\
\text { staff's sense of } \\
\text { competence }\end{array}$ & $\begin{array}{c}\text { Baseline (before } \\
\text { intervention) Follow-up } \\
\text { week } 12 \text { and } 24 .\end{array}$ \\
\hline
\end{tabular}


Citation: Torres-Castro S, López-Ortega M, Martínez-Ruiz A, et al. Pilot study and key feasibility factors of a staff training intervention and reduction of antipsychotic prescription practice in Mexican urban care homes: study protocol. J Ment Health Aging. 2018;2(1):47-55.

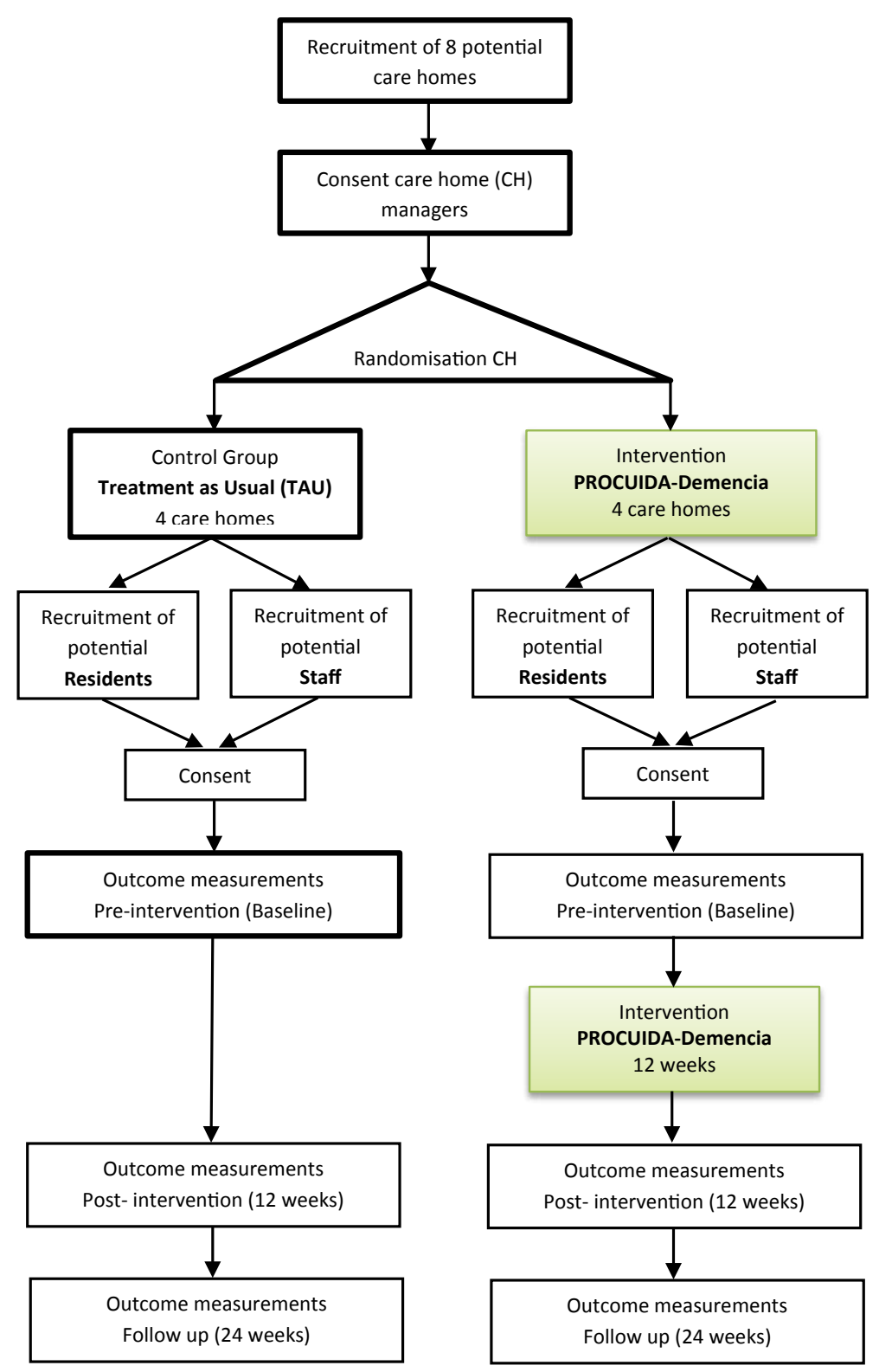

Figure 1. Study Diagram.

in terms of research questions for staff, residents and relatives in a conceptual explanation of what is happening in the study.

\section{Intervention fidelity}

Fidelity of the intervention, all the staff training sessions in the intervention care homes will be video recorded. In addition, each manager will organize an agenda for staff to complete activity logs with type and frequency scheduled for each therapy: Therapeutic Lies Log, Personcentred Environment Assessment Matrix, Interventions Log (Psychomotor Dance Therapy attendance sheet; Antecedent-Behavior-Consequence (ABC); Psychological Formulations), staff turnover and sickness logs. The Mexican principal investigator will supervise PROCUIDA-Demencia care homes, every week for two hours to monitor adherence.

\section{Care home inclusion/exclusion criteria}

Each care home was chosen with the following criteria:
1) A $20 \%$ of residents suspected with a mild to moderate dementia diagnosis (DSM-V criteria) (Clinical Dementia Rating/CDR); 2) Care Homes that have not had similar training on Psychosocial Interventions or that are currently reviewing Antipsychotic Medication; 3) Care Homes with at least one spacious and ventilated room designated for PROCUIDA-Demencia Study activities; 4) Care Homes with manager and proprietor willing to be involved in the program and allow staff to take part in the training; 5) Psychologist and GP to support residents in case of a crisis; 6) Health and safety protocols in place.

\section{Participant's inclusion/exclusion criteria}

Residents living permanently in the care home (not respite); age $60+$ and above. INGER has a number of Old Age Psychiatrists that will collaborate with us to confirm dementia and stage diagnosis. Inclusion Criteria: 1) Dementia diagnosis (DSM V criteria); 2) SPPB screening 
measure, cut off point 10 to prevent risk of falls; 3) Signed informed consent; 4) Participants must speak and understand Spanish, alternative English consent forms will be given if the participant is not Spanish-native speaker; 5) Preferably on current antipsychotic medication prescription. Relatives will be encouraged by staff to volunteer in the PROCUIDADemencia activities. The aim is to involve relatives in the personalized care of their family member taking part in the study. Relatives need to 1) Consent to participate in the PROCUIDA-Demencia activities organized by the care home; 2) Consent to participate in the Focus Groups targeting family members at the end of the study; 3) Visit at least once a month, in order to witness the staff-resident intervention activities; 4) Read/Speak Spanish. See Figure 1 with the study diagram.

\section{Care staff inclusion/exclusion criteria}

1) Consent to participate in the PROCUIDA-Demencia study; 2) Available for the three day staff training, which will be delivered by the PI's to ensure that the interventions are being implemented in the care homes; 3) Commitment to support the study whether or not allocated to intervention or control group for 24 weeks. Staff those will be willing to facilitate musical and dancing sessions. It will not require professional dance/musical experience, but a rhythmic response to music will be required.

\section{Outcome measures}

We will collect demographic data throughout a questionnaire that will examine the sample population age, education, gender, ethnicity, previous occupation, and conditions of their transition to institutional care. Staff questions will address previous training on dementia care and overall care qualifications. In addition, standardized and non-standardized outcome measures will be applied for a preliminary understanding of any behavioral, mood and cognitive changes before a larger controlled trial. See Table 1 with a description of each measure.

\section{Adverse Event}

In case of any adverse event, such as a fall, or hospitalization as a result of participating in the intervention, the principal investigator in Mexico will report and monitor participants recovery. In case of attrition, the researcher will report if this was due to intervention participation, natural causes or health deterioration. The investigator will call by telephone the control group care homes (TAU) on a weekly basis. This is to ensure that there are no logistical problems affecting participants' continued participation and inquire about any significant health changes.

\section{Data Management and analysis}

Data collection of the outcome measures outlined above will be conducted by blinded research assistants recruited in Mexico. Adverse Events (if applicable) and Care Home logs will be monitored by Mexican PI to prevent un-blinding research assistants.
Quantitative data management and analysis: Quantitative data will be cleaned, coded and entered into STATA SE 11 by trained Research Assistants. Data missing at follow-up will be handled using the 'last observation carried forward' method with baseline scores being carried through to follow-up. Data accuracy will be checked manually and assessed by examining the frequency tables to identify any potentially incorrect entries. Distribution will be assessed using histogram with a normal curve charts.

Staff demographic characteristics will be summarized using descriptive statistics. Inferential statistics, such as paired-sample t-tests and one-way between-groups ANOVAs, will be used to identify any statistically significant differences over time for the total sample, and between selected sub groups, for example: participating care homes; staff who received the PROCUIDA-Demencia training and those who did not. A power analysis will be conducted to calculate the sample size necessary to detect an effect of intervention for the full RCT.

The Review of Antipsychotic Medication (RAM) will be undertaken with consent from the participant or the next of kin if applicable. The medical team of this study is qualified to review antipsychotic medication and monitor the implementation of psychosocial interventions to reduce BPSD.

Previous Psychomotor Dance Therapy studies have not reported adverse effects [33] noted that only one participant with dementia who only attended $50 \%$ of the study showed a bit of frustration for not being as graceful as when he was younger owing breathing difficulties [33]. Nevertheless this participant recommended the DANCIN model. Participants taking part as dancers will be required to wear sturdy sole shoes in a ventilated and spacious room in the care home, beverages will be provided to prevent dehydration.

Doll Therapy and Reminiscence Therapy have no known side effects, overdose-risk or drug interactions, and no prescription or clinic visits necessary, therefore is easy to use and safe.

In addition, the assessment of associated costs will provide valuable inputs for future planning of public or private services for older adults with dementia in Mexico.

Qualitative data management and analysis: Focus Group is a research method that explores and clarifies participants' views in ways that would be less easily accessible in semi-structured individual interviews [34,35]. Purpose sampling will be conducted in the care homes taking part in PROCUIDA-Demencia group. Focus Groups will be conducted with staff; residents and relatives to obtain qualitative data regarding the acceptance and feasibility of PROCUIDA-Demencia on staff care practice and staffresident interaction. The Focus Groups will be conducted after the intervention phase, at times convenient with the care home manager and in a quiet, comfortable room with no disturbances. Six Focus Groups will be conducted in 
Citation: Torres-Castro S, López-Ortega M, Martínez-Ruiz A, et al. Pilot study and key feasibility factors of a staff training intervention and reduction of antipsychotic prescription practice in Mexican urban care homes: study protocol. J Ment Health Aging. 2018;2(1):47-55.

the PROCUIDA-Demencia Care Homes as follows: i) Two groups with staff, each with six to ten participants for approximately 90 minutes, including those who have and have not participated in PROCUIDA-Demencia Model. ii) Two groups with residents, one with a maximum of ten residents who are more able to communicate/express their views, and one with a maximum of residents who need more time and support to communicate their views, each lasting for approximately 60 minutes. iii) Two groups with relatives, each with six to ten participants for approximately 60 minutes.

The Focus Group interview schedule will include open questions about the feasibility of PROCUIDA-Demencia on training the staff and residents (for example changes before, during and after the training). Relatives' questions will address specific areas such as noticed changes of staff attitudes to care (for example more engaged, more distracted), describe residents participation during the training. Data collected from Focus Groups will be recorded with a goodquality digital audio recorder.

Regulatory and management issues: This study involves five work packages: Development of the Staff Training package (PROCUIDA-Demencia) for the Mexican care homes, staff training, intervention, data analysis, and publications. Research assessments will be completed using paper-copy questionnaires, using only code numbers as identifiers for participants. Data and schedules for each care home will be securely stored and electronic data will be password protected. All data will be stored using a linked anonymised system so that no personal identifiers are present on the schedules or databases, including the collected study data. Anonymised data will be securely transferred to INGER who are responsible for data management and data analysis and operate strict data protection protocols.

\section{Interventions}

The PROCUIDA-Demencia study aims to train staff in the following three areas: 1) Understanding Behavior that challenges and Review of Antipsychotic Medication; 2) Person-Centred Care Therapy and 3) A set of Psychosocial Interventions such as: Psychomotor Dance Therapy, Doll Therapy Intervention and Reminiscence Group Therapy. See Figure 2 for a PROCUIDA-Demencia Interventions diagram

\section{Understanding behavior that challenges and Review of Antipsychotic Medication (RAM)}

Understanding Stress and Distress is a priority in every day care. Organizations require strategies to reduce challenging behaviors. Based on the Newcastle Model to understand Behavior [36] PROCUIDA-Demencia will provide training on how to understand certain behavioral profiles, from probable physical pain, environment impact to side effects of medication. The training will cover elements of the Antecedent-Behavior-Consequence approach to quantify the behaviors frequency. Additionally, information by the Alzheimer's Society UK such as the optimizing antipsychotic prescription will be addressed with participants.

\section{Person-Centre Care Therapy (PCC)}

Studies have found that a positive attitude towards people with dementia, and stronger intentions to implement person-centred care strategies, predicted a greater sense of competence to provide care [37]. PROCUIDA-Demencia will encourage attitudes of personalized care, and will provide training to care home staff to develop personcentred care plans, implement a positive and person-centred communication style. The training manual will include activities suggested by the person-centred communication approach [38] to reflect and acquire skills to improve care. Person-Centred Therapy has been described as 'relationship therapy' and provides theory and practice in the context of power organization and clients, the development of self and personhood [39]. In the context of Dementia, it was Tom Kit wood who addressed the 'malignant social psychology' and the need to meet individual needs of institutionalized people living with dementia.

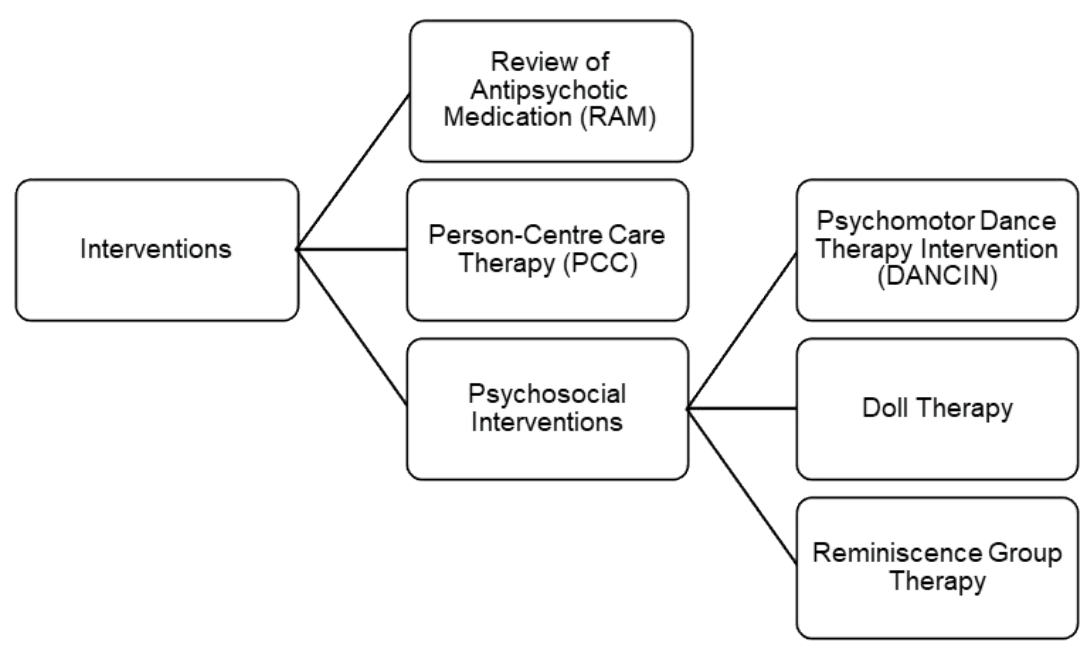

Figure 2. PROCUIDA-Demencia Interventions Diagram. 


\section{Psychosocial interventions}

Psychomotor Dance Therapy Intervention (DANCIN) was based on the Psychomotor Therapy framework and dancing by UK partner. This is a validate model by a Physiotherapist, Clinical Psychologist and Old Age Psychiatrist at Newcastle University.

Furthermore, the current study applies Psychomotor Therapy, which is widely practiced in other countries such as the Netherlands to improve the health of older people [40], and a core approach for improving residential Dementia care [Objective 5, Measure No. 17 of the French 'Plan Alzheimer's' (2008-2012)]. Psychomotor Therapy involves three dimensions: i) Motor (balance, fast/slow interpersonal coordination, hand-grip, gesture and facial expression); ii) Emotional-Affective (expressing feelings, verbal and non-verbal communication); and iii) Cognitive (attention and planning movement in space; reflecting on personal performance), with the aim of using movement activities and paying attention to bodily experiences [40-42]. The approach is usually complemented by hand by hand touch, relaxation and breathing techniques [43]. Additionally, arm-chair exercises to warm-up and cool down, and four simplified choreographies are included for staff to repeat with residents. DANCIN has shown positive effect on behavior that challenges, such as agitation, anxiety, insomnia and selfesteem $[44,45]$.

Doll Therapy, applied in people with dementia, it has been associated with a number of benefits which include a reduction in episodes of distress, an increase in general well-being, improved dietary intake and higher levels of engagement with others [46]. Doll Therapy remains controversial and may remain as inappropriate when applied to the concepts of dignity (people with dementia are encouraged to interact with dolls) and non-malfeasance (potential distress this therapy could cause for family members). In this perspective, people with dementia will decide if they want a doll or not. This is in line with the United Nations Convention on the Rights of Persons with Disabilities, which provides a legal framework that considers the person with dementia as a 'rights holder' and places them at the centre of any ethical dilemma. In addition, those with responsibility towards caring for people with dementia have their capacity built to respect, protect and fulfill dementia patient's rights and needs [47]. Residents generally support the use of dolls, believing that dolls can have a positive impact on their well-being.

Reminiscence Group Therapy literature reports have found that Reminiscence Therapy improves cognitive functions and mood in people with Dementia [48]. Psychological interventions, whether directed at cognitive functioning or emotional expression, are frequently offered on a group basis to people with Dementia. In addition, it will be important to enable participants and their family members to play a role in determining what interventions are relevant and acceptable, and what outcomes are desirable [49]. Subramaniam et al. found that after a Life Story Book (LSB) session with relatives and care staff, quality of relationship as rated by relatives improved significantly $(2,39)=19.37, \quad \mathrm{p}<0.001)$ and staff knowledge regarding the resident and attitudes to dementia improved [50]. The creation of LSBs either through a life review process or by relatives without involving the person has benefits for people with dementia, relatives and staff in care homes.

\section{Ethics Approval}

Ethical approval is granted by the School of Health in Social Science, University of Edinburgh, reference number STAFF068 and Instituto Nacional de Geriatria, reference number PI-011/2016.

\section{Consent}

Consent to participate and data collection will be taken by research assistants from Mexico.

The datasets generated and/or analyzed during the current study will not be publicly available but available from the corresponding author on reasonable request.

\section{Trial Status}

The trial started recruitment in January 2017 and was complete follow up assessments in August 2017. Results will be reported in other article.

\section{Acknowledgements}

STC is the site lead for the trial and principal investigator. AG is the chief investigator of PROCUIDA-Demencia study. STC, MLO and AG were involved in design, fellowship application, and protocol development. MLO provides statistical expert advice. AM provides specialist medical advice and supervision of antipsychotic medication practice in participating care homes. LMG have provided expert advice in older adult health and ageing process, including $\mathrm{AD}$ and dementia, as well as in the development of the study protocol. All authors read and approved the final manuscript.

Funding: PROCUIDA-Demencia is funded by the Newton British Academy Fellowship awarded to STC and AG. The views expressed are those of the authors and not necessarily those of the British Academy or the Department of Health in UK or Mexico.

We thank to the following academics for comments on early drafts of this feasibility study:

Dr Luis Miguel Gutierrez (INGER Director); Dra Carmen García (INGER, Research Director); Dr Matthew Prina (Lecturer in Ageing \& Mental Health, Health Services \& Population Research-King's College London); Prof. Ian James (Clinical Psychologist at Bradford University \& lead of Challenging Behavior Team at Northumberland NHS Trust); Prof. Charlotte Clarke (Head of the School of Health in Social Sciences, University of Edinburgh). 
Citation: Torres-Castro S, López-Ortega M, Martínez-Ruiz A, et al. Pilot study and key feasibility factors of a staff training intervention and reduction of antipsychotic prescription practice in Mexican urban care homes: study protocol. J Ment Health Aging. 2018;2(1):47-55.

\section{Competing Interest}

The authors declare that they have no competing interests.

\section{References}

1. Prince M, Wimo A, Knapp M, et al. World Alzheimer Report. Alzheimer's Disease International. London. 2015.

2. Prince M, Comas-Herrera A, Knapp M, et al. World Alzheimer Report. Alzheimer's Disease International. London. 2016.

3. National Institute for Health and Clinical Excellence/ Social Care Institute for Excellence: Dementia: the NICE SCIE guideline on supporting people with dementia and their carers in health and social care. National Collaborating Centre for Mental Health. London. 2007.

4. Instituto Nacional de Geografía y Estadística. Estadísticas a propósito del día internacional de las personas de edad. Datos Nacionales. México. 2016.

5. Llibre JJ, Ferri C, Acosta D, et al. Prevalence of dementia in Latin America, India, and China: a population-based cross-sectional survey. Lancet. 2008;372:464-74.

6. Mejia-Arango S, Gutierrez LM. Prevalence and incidence rates of dementia and cognitive impairment no dementia in the Mexican population: data from the Mexican Health and Aging Study. J Aging Health. 2011;23:50-74.

7. López Ortega M, Jiménez-Bolón JE. Dependencia y Cuidados de Largo Plazo. In: Gutiérrez-Robledo LM, García-Peña MC, Jiménez-Bolón JE. (Eds.). Envejecimiento y Dependencia. Realidades y Previsión para los Próximos años México, DF: Academia Nacional de Medicina. 2014;pp29-62.

8. Gutiérrez-Robledo LM, Arrieta I. Plan de acción Alzheimer y otras demencias, México 2014. Accessed 27 June 2017.

9. Fossey J, Ballard C, Juszczak E, et al. Effect of enhanced psychosocial care on antipsychotic use in nursing home residents with severe dementia: cluster randomised trial. Br Med J. 2006;332:756-8.

10. Goyder J, Orrell M, Wenborn J, et al. Staff training using STAR: a pilot study in UK care homes. Int Psychogeriatr. 2012;24(6):911-20.

11. Whitaker R, Ballard C, Stafford J, et al. Feasibility study of an optimised person-centred intervention to improve mental health and reduce antipsychotics amongst people with dementia in care homes: study protocol for a randomised controlled trial. Trials. 2013;14:13.

12. Ballard C, Hanney ML, Theodoulou M, et al. The dementia antipsychotic withdrawal trial (DART-AD): long-term follow-up of a randomised placebo-controlled trial. Lancet Neurol. 2009;8:151-7.

13. Ballard C, Orrell M, YongZhong S, et al. Impact of antipsychotic review and non pharmacological intervention on antipsychotic use, neuropsychiatric symptoms, and mortality in people with dementia living in nursing homes: A factorial cluster-randomized controlled trial by the Well-Being and Health for People with Dementia (WHELD) program. Am J Psychiatry. 2016;173(3):252-62.

14. Gentry M, Iceton J, Milne D. Managing challenging behaviour in the community: methods and results of interactive staff training. Health Soc Care Community. 2001;9:143-50.

15. Beattie E, O'Reilly M, Moyle W, et al. Multiple perspectives on quality of life for residents with dementia in long term care facilities: protocol for a comprehensive Australian study. Int Psychogeriatr. 2015;27(10):1739-47.

16. Jessop T, Harrison F, Cations M, et al. Halting Antipsychotic Use in Long-Term care (HALT): a singlearm longitudinal study aiming to reduce inappropriate antipsychotic use in long-term care residents with behavioural and psychological symptoms of dementia. Int Psychogeriatr. 2017;29(8):1391-403.

17. Brooker DJ, Latham I, Evans SC, et al. FITS into practice: translating research into practice in reducing the use of anti-psychotic medication for people with dementia living in care homes. Aging Ment Health. 2016;20(7):709-18.

18. Froggatt K, Parker D. Care homes and long-term care for people with dementia. In: Hughes JC, Lloyd-Williams M, Sachs GA. (Eds.) Supportive care for the person with dementia. Oxford: Oxford University Press. 2010;pp181-18.

19. Nelson S, Wild D, Szczepura A. Innovation in residential care homes: an in reach nursing team project. Primary Health Care. 2009;19:31-3.

20. Wild D, Szczepura A, Nelson S. Residential care home workforce development: The rhetoric and reality of meeting older residents' future care needs, Joseph Rowntree Foundation. 2010. Accessed 27 June 2017.

21. Möhler R, Köpke S, Meyer G. Criteria for reporting the development and evaluation of complex interventions in healthcare: revised guideline (CReDECI 2). Trial. 2015;3(16):204.

22. Russell D, Hoare ZSJ, Whitaker R, et al. Generalised method for adaptive randomisation in clinical trials. Stat Med. 2011;30:922-34.

23. Ritchie J, Spencer L. Qualitative data analysis for applied policy research. In: Bryman A, Burgess RG. (Eds.) Analysing qualitative data. London: Routledge; 1994;pp173-94.

24. Gale NK, Heath G, Cameron E, et al. Using the framework method for the analysis of qualitative data in multidisciplinary health research. BMC Med Res Methodol. 2013;13:117. 
25. Guralnik JM, Simonsick EM, Ferrucci L, et al. A short physical performance battery assessing lower extremity function: association with self-reported disability and prediction of mortality and nursing home admission. J Gerontol. 1994;49:M85-94.

26. Morris JC. The Clinical Dementia Rating (CDR): current vision and scoring rules. Neurology. 1993;43:2412-4.

27. Rosas-Carrasco O, Torres-Arreola LP, Guerra-Silla MG, et al. Validation of the Quality of Life in Alzheimer's Disease (QOL-AD) scale in Mexican patients with Alzheimer, vascular and mixed-type dementia. Rev Neurol. 2010;51(2):72-80.

28. Matias-Guiu JA, Fernandez de Bobadilla R, Escudero G, et al. Validation of the Spanish version of Addenbrooke's Cognitive Examination III for diagnosing dementia. Neurología. 2015;30(9):545-51.

29. Boada M, Tárraga L, Modinos G, et al. Neuropsychiatric Inventory-Nursing Home version (NPI-NH): validación española. Neurología. 2005;20:665-73.

30. Maslach C. The measurement of experienced burnout. J Occupational Behav. 1981;2:99-113.

31. Lintern T, Woods B. Approaches to dementia questionnaire. Bangor: University of Wales. 2001.

32. Schepers AK, Orrell M, Shanahan N, et al. Sense of competence in Dementia Care Staff (SCIDS) Scale: development, reliability and validity. International Psychogeriatrics. 2012.

33. Guzmán A, Freeston M, Hughes J, et al. Psychomotor Dance Therapy Intervention (DANCIN) for people with dementia in care homes: a multiple-baseline single case study. Int Psychogeriatr. 2016;10:1695-715.

34. Kitzinger J. Chapter 3: Focus groups. In: Pope C, Mays N. (Eds.) Qualitative research in health care. Malden MA: Blackwell. 2006;pp21-31.

35. Green J, Thorogood N. Qualitative methods for health research. London: Sage Publications. 2005;126-8.

36. James I. Understanding behaviour in dementia that challenges: a guide to assessment and treatment. London: Jessica Kingsley Publishers. 2011;105-46.

37. Mullan MA, Sullivan KA. Positive attitudes and personcentred care predict of sense of competence in dementia care staff. Aging Ment Health. 2016;20:407-14.

38. Motschnig R, Nykl L. Person-centred communication, theory, skills \& practice Berkshire: open university press. 2009.

39. Tudor K, Worrall M. Person-centred therapy a clinical philosophy. Advancing Theory in Therapy. East Sussex: Routledge. 2006.

40. Dröes RM. Psychomotor group therapy for demented patients in the nursing home. In: Miesen MLB, Jones MMG. (Eds.) Care-giving in dementia research and applications. London: Routledge. 1997;2:pp95-118.

41. Calmels D. ¿Qué es Psicomotricidad? Los trastornos psicomotores y la prácticapsicomotriz [What is psychomotor practice? Psychomotor Disorders and Practice]. Buenos Aires: Lumen. 2003.

42. Probst M, Knapen J, Poot G, et al. Psychomotor therapy \& psychiatry: what's in a name? The Open Complementary Medicine Journal. 2010;2:105-13.

43. Camacho M, Paolillo G. Relajación y narración: recursos originales en la práctica del psicomotricista en educación. Revista Iberoamericana de Psicomotricidad y Técnicas Corporales. 2004;15:55-73.

44. Guzmán-García A, Hughes JC, James IA, et al. Dancing as a psychosocial intervention in care homes: a systematic review of the literature. Int $\mathrm{J}$ Geriatr Psychiatry. 2013;28:914-24.

45. Guzmán-García A, Mukaetova-Ladinska E, James IA. Introducing a lesson of Latin ballroom dance class to people with dementia living in care homes, benefits and concerns: a pilot study. Dementia. 2013;12:523-35.

46. Mitchell G, Templeton M. Ethical considerations of doll therapy for people with dementia. Nurs Ethics. 2014;21:720-30.

47. Alander H, Prescott T, James IA. Older adults' views and experiences of doll therapy in residential care homes. Dementia. 2015;14:574-88.

48. Cotelli M, Manenti R, Zanetti O. Reminiscence therapy in dementia: A review. Maturitas. 2012;72:203-5.

49. Scott J, Clare L, Woods B. Do people with dementia benefit from psychological interventions offered on a group basis? Clin Psychol Psychother. 2003;20:186-96.

50. Subramaniam P, Woods B, Whitaker C. Life review and life story books for people with mild to moderate dementia: a randomised controlled trial. Aging Ment Health. 2014;18:363-75.

\section{*Correspondence to:}

Sara Torres-Castro

Instituto Nacional de Geriatría, Mexico City, Mexico

E-mail: saratorrescastro@gmail.com

Tel: +52 5513414923 